\title{
28 Research Soure \\ Soft Tissue Deformation Modeling in the Procedure of Needle Insertion: A Kriging-based Method
}

\section{Yong Lei}

Zhejiang University https://orcid.org/0000-0003-0235-5203

\section{Murong Li}

Zhejiang University

Dedong Gao ( $\nabla$ gaodd@zju.edu.cn )

Qinghai University https://orcid.org/0000-0002-4732-5916

\section{Original Article}

Keywords: Kriging metamodel, needle insertion, real-time simulator, soft tissue deformation

Posted Date: August 2nd, 2020

DOl: https://doi.org/10.21203/rs.3.rs-50251/v1

License: (1) This work is licensed under a Creative Commons Attribution 4.0 International License.

Read Full License 


\section{RESEARCH}

\section{Soft Tissue Deformation Modeling in the Procedure of Needle Insertion: A Kriging-based Method}

\author{
Yong Lei ${ }^{1}$ \\ , Murong $\mathrm{Li}^{1}$ \\ and Dedong $\mathrm{Gao}^{2 *}$ \\ ${ }^{*}$ Correspondence: \\ gaodd@zju.edu.cn \\ ${ }^{2}$ School of Mechanical Engineering \\ Qinghai University, Xining, 810016 \\ China \\ Full list of author information is \\ available at the end of the article
}

\begin{abstract}
The simulation and planning system (SPS) requires accurate and real-time feedback regarding the deformation of soft tissues during the needle insertion procedure. Traditional mechanical-based models such as the finite element method (FEM) are widely used to compute the deformations of soft tissue. However, it is difficult for the FEM or other methods to find a balance between an acceptable image fidelity and real-time deformation feedback due to their complex material properties, geometries and interaction mechanisms. In this paper, a Kriging-based method is applied to model the soft tissue deformation to strike a balance between the accuracy and efficiency of deformation feedback. Four combinations of regression and correlation functions are compared regarding their ability to predict the maximum deformations of ten characteristic markers at a fixed insertion depth. The results suggest that a first order regression function with Gaussian correlation functions can best fit the results of the ground truth. The functional response of the Kriging-based method is utilized to model the dynamic deformations of markers at a series of needle insertion depths. The feasibility of the method is verified by investigating the adaptation to step variations. Compared with the ground truth of the finite element (FE) results, the maximum residual is less than $0.92 \mathrm{~mm}$ in the $Y$ direction and $0.31 \mathrm{~mm}$ in the $X$ direction. The results suggest that the Kriging metamodel provides real-time deformation feedback for a target and an obstacle to a SPS.
\end{abstract}

Keywords: Kriging metamodel; needle insertion; real-time simulator; soft tissue deformation

\section{Introduction}

In needle-based minimally invasive surgeries, needle insertion errors always lead to a large probability of serious complications and increase the physical pain of patients [1-4]. Real-time feedback regarding soft tissue deformation during needle insertion, especially in the region around the target and obstacles, and the experience of the attending physicians affect the insertion accuracy [4]. The simulation and planning system (SPS) can provide pre- and intraoperative deformation information and help physicians to sharpen their skills [5], which can effectively improve the success rate of minimally invasive surgeries. Soft tissue visualization during the needle insertion procedure, which is the most important part of the SPS, is realized by a deformation model of the soft tissue [6]. 
The soft tissue deformation model has been widely studied in the literature [7-11], of which continuum mechanical-based modeling methods such as the finite element method (FEM) are the most popular [11-13]. Dimaio et al. established the soft tissue of a simple geometry using the FEM, in which the refined elements along the needle path were configured and the interaction force was experimentally loaded onto the node path. The material properties of the tissue were modeled as the linear elasticity [13]. Misra et al. [14] reviewed and sorted out the tool-tissue interaction models based on the tissue material properties, which revealed that the hyperelastic model had a relatively high accuracy. Kobayashi et al. modeled the nonlinear and viscoelastic liver based on the finite element method [15]. The complicated organ geometry and boundary conditions were taken into account in $[16,17]$. The complex boundary conditions and geometry of real organs, as well as the viscoelastic and heterogeneous material properties, increased the computational complexity.

Furthermore, the needle insertion process includes material failure and a new internal surface formation; thus, the underlying physics is also an essential part. Some of the traditional FEM modeling methods, although able to provide fast calculations of tissue deformation, lead to unpleasing visual results during the needle insertion procedure due to their rough simplification of the needle puncture process. Takabi [18] presented a comprehensive survey mainly on the modeling of tissue cutting, focusing on the mechanical concepts and modeling techniques. Gokgol et al. [19] modeled the FEM cutting process by defining a fracture work, the nodal in the FEM model break when the cutting energy exceeded the fracture work. The thresholds can also be other failure parameters such as the maximum shear strain [20]. Oldfield and Dini used cohesive elements in the ABAQUS commercial software to describe the material failure [21]. However, all of these methods require the elements along the needle path to be sufficiently small, which may result in a large computational burden and instability in the calculations. Recently, the extended finite element method (X-FEM), typically utilized for crack propagation, was also applied to soft tissue cutting [22]. It reserved the initial mesh topology without very high-resolution grids in modeling the discontinuities, but an appropriate enrichment function was difficult to establish. To eliminate the mesh dependence in the traditional FEM, the meshless method was proposed for soft tissue deformation modeling, which is particularly suitable for simulating large deformation and cutting processes since it is not required to calculate equations on the grid [23,24]. However, the basis interpolation function in the meshless method is complex and consumes too many computing resources.

A successful surgery simulation and planning system requires two contradictory basic processes: physical reality and real-time dynamic interaction. However, most existing needle insertion deformation models with high accuracy during needle insertion are simulated offline. It is difficult for the FEM or other methods to balance the acceptable image fidelity and real-time deformation feedback, which limits their applications in real clinical trainings $[25,26]$. In this paper, a Kriging metamodel is applied to model and predict the deformation of obstacles and targets inside soft tissue during the needle insertion procedure. The Kriging metamodel was addressed by the South African mining engineer Krige and developed by Matheron, a French mathematician [27]. It is a simplified computer-based simulation model that has 
an input/output function based on the surface response [28]. The advantages of the Kriging metamodel are twofold: First, the Kriging metamodel can reduce the large requirement for computing resources and offer real-time simulation, especially being able to overcome the challenges of computational techniques in complex simulation models [29]. Hence, depending on the accurate simulation dataset generated by other offline needle-tissue interaction models, the online Kriging model can be established rapidly without reducing the accuracy of the original model by much. Second, the computer-based simulation program is deterministic, that is, the same input corresponds to the same output, which cannot fully reflect the uncertainty of mechatronic systems [30,31]. In robot-assisted needle insertion systems, complex factors such as the imaging equipment, physician's skill and patient's condition lead to uncertainty regarding tissue deformation. The Kriging metamodel with the random sampling method can reflect the uncertainties of robot-assisted needle-tissue interactions.

In our work, the input dataset is generated by Latin hypercube sampling (LHS), the parameters of which include the material properties of the tissues and needle, the geometrical properties of the needle and the solver parameters. Ten characteristic markers are selected to represent the targets and obstacles inside the tissue body. The output dataset is generated by running the needle-tissue coupling FE model presented in [32], which is used as the ground truth of the Kriging-based model. The validity and feasibility of the proposed Kriging-based model are analyzed, and the results suggest that the combination of the Kriging metamodel and the highprecision finite element model provides real-time deformation feedback for a target and an obstacle to the SPS.

The rest of this paper is organized as follows. Section II introduces the data preparation, the basic theory of the Kriging model and the maximum deformation modeling of needle insertion at a fixed depth. In Section III, the functional-based Kriging model is applied to establish the deformation model at a series of depths, of which the adaptation to the step variation is also analyzed. Section IV discusses the proposed Kriging model. Finally, conclusions and future work are given in Section $\mathrm{V}$.

\section{Flowchart of Kriging Deformation Online Prediction}

A flowchart of Kriging online deformation prediction is shown in Fig.1. In offline process, a physic-based model, such as finite element based needle insertion model is utilized to generate dataset. The dataset include the input parameters and output deformations in different locations. The input parameters include material parameters of soft tissues, needle parameters and other parameters affecting the deformation results obviously. The dataset is provided and offline trained for Kriging meta model. Training process is for tuning Kriging parameters to best fit the deformation prediction model. Kriging model is in essence a computational model; thus, it can realize real-time prediction while maintain the accuracy of the physic-based model. In surgery simulation and planning, when input current parameters of tissue and needle, the Kriging model can online predict soft tissue deformation for surgery training or needle path planning. 


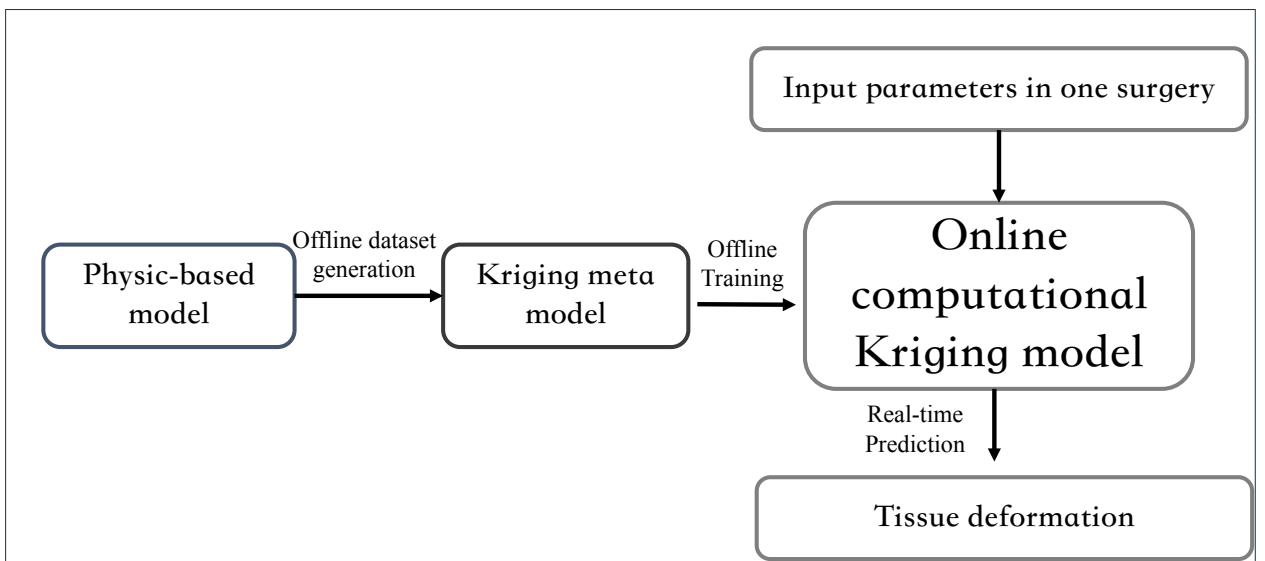

Figure 1: Flowchart of Kriging online prediction

\section{Deformation Modeling of Needle Insertion at a Fixed Depth}

A finite element model for the needle-tissue interaction was built by our group to simulate tissue deformation [32]. In this paper, we aim to predict the motion of the target and obstacles inside the tissue in real time. To test the validation of the Kriging-based prediction model, ten characteristic markers in the FE model are selected as the observation points, as shown in Fig. 2. The observation points are chosen according to the distance from the needle body. The points near the needle body include $\mathrm{N} 1(-0.0064,-0.0256), \mathrm{N} 2(0.0052,-0.0238), \mathrm{N} 3(-0.0058,-0.0445)$, N4 $(0.0054,-0.0447)$, N5 $(-0.0068,-0.0629)$ and N6 $(0.0041,-0.0641)$, which represent locations near the needle but at different insertion depths, wherein points 1,3 , and 5 are distributed on the left side of the needle body and 2,4 , and 6 are distributed on the right side. They are not symmetrical about the needle body even though they are distributed bilaterally, as the needle will bend as the insertion depth into the soft tissue increases due to the beveled needle tip, as shown in Fig. 2. Observation points N7 $(-0.0254,-0.0232)$, N8 $(-0.0485,-0.0228)$, N9 $(-0.0290,-0.0637)$ and N10 $(-0.0501,-0.0649)$ are far from the needle body, wherein 9 and 10 are also near the fixed boundaries.

There were 11 variables chosen and listed in Table 1, wherein x1, x2, and x11 are the material parameters of soft tissues, $\mathrm{x} 3-\mathrm{x} 7$ are the needle parameters, $\mathrm{x} 8$ - $\mathrm{x} 9$ are the FEM solver parameters and $\mathrm{x} 10$ is the friction coefficient between the needle and soft tissue.

The input variables are denoted as $X=\left[x_{1}, \cdots, x_{11}\right]$, which are employed to construct the design matrix of the Kriging-based model. Running the finite element model 20 times, the displacements at the 10 observed locations are collected as the output responses of the Kriging model, denoted by $Y=\left[u_{x y}^{1}, \cdots, u_{x y}^{10}\right]^{T}=$ $\left[y_{1}, y_{2}, \cdots, y_{10}\right]^{T}, y_{i} \in \mathbf{R}^{20}$. The soft tissue deformation occurs in both the $x$ and $y$ directions in the two-dimensional case; hence, the output response $u_{x y}$ is the resultant displacement in both directions and is written as

$$
u_{x y}=\sqrt{u_{x}^{2}+u_{y}^{2}}
$$




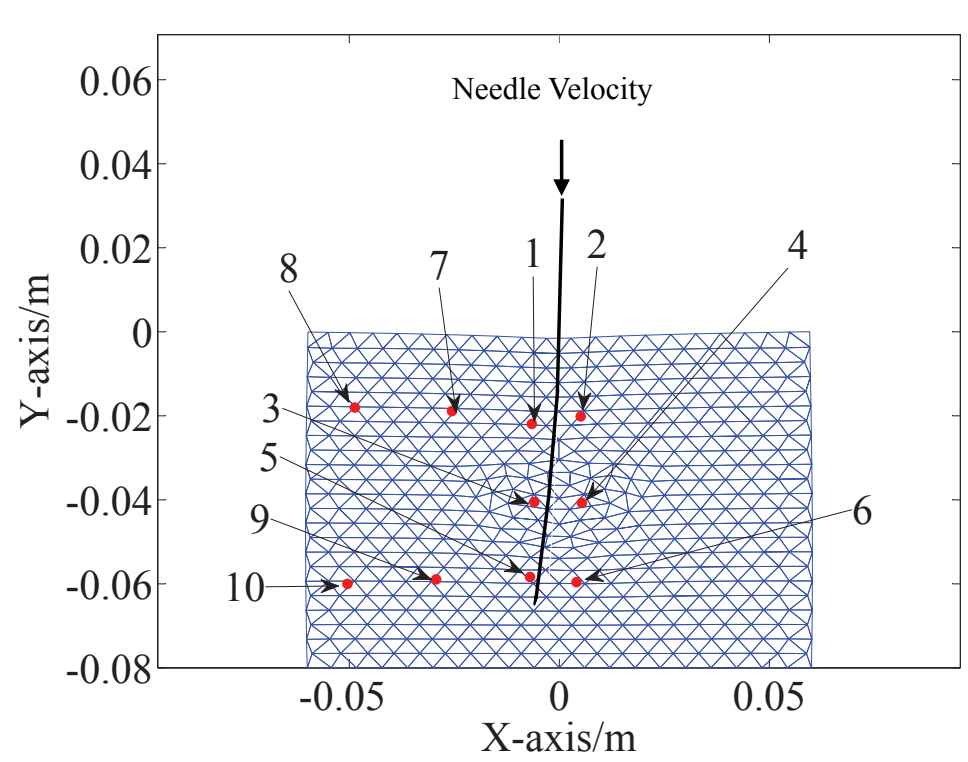

Figure 2: 10 observation points in the needle insertion procedure for the Kriging-based model

Table 1: Factors and range of values in the soft tissue deformation experiment

\begin{tabular}{clcc}
\hline \multirow{2}{*}{ Variable } & Factor & \multicolumn{2}{c}{ Range } \\
\cline { 3 - 4 } & & Maximum Value $x_{L}$ & Minimum value $x_{U}$ \\
\hline$x_{1}$ & Young's modulus of the soft tissue & $10 \mathrm{KPa}$ & $50 \mathrm{KPa}$ \\
$x_{2}$ & Soft-tissue Poisson ratio & 0.3 & 0.5 \\
$x_{3}$ & Tip angle & 0 & $90^{\circ}$ \\
$x_{4}$ & Needle penetration angle & $-15^{\circ}$ & $15^{\circ}$ \\
$x_{5}$ & Needle radius & $0.2 \mathrm{~mm}$ & $0.6 \mathrm{~mm}$ \\
$x_{6}$ & Needle length & $100 \mathrm{~mm}$ & $250 \mathrm{~mm}$ \\
$x_{7}$ & Young's modulus of the needle & $200 \mathrm{GPa}$ & $300 \mathrm{GPa}$ \\
$x_{8}$ & Rayleigh coefficient of damping $\alpha$ & 0.01 & 0.1 \\
$x_{9}$ & Rayleigh coefficient of damping $\beta$ & 0.01 & 0.1 \\
$x_{10}$ & Coefficient of friction & $30 \mathrm{~N} / \mathrm{m}$ & $50 \mathrm{~N} / \mathrm{m}^{2}$ \\
$x_{11}$ & Area density & $20 \mathrm{~kg} / \mathrm{m}^{2}$ & $30 \mathrm{~kg} / \mathrm{m}^{2}$ \\
\hline
\end{tabular}

Kriging model construction

The Kriging prediction model is an interpolation of known observation locations, of which the mean square error equals zero. The Kriging model is usually expressed as a combination of a polynomial and its deviation [33], written as

$$
\hat{y}(x)=f^{T}(x) \beta^{*}+r^{T}(x) \gamma^{*},
$$

where $f(x)$ is the basis polynomial function, usually a regression function. $r(x)$ is the column vector of correction matrix $\mathcal{R}_{m \times m}=\left[R\left(x_{i}, x_{1}\right), R\left(x_{i}, x_{2}\right), \cdots, R\left(x_{i}, x_{m}\right)\right]^{T}, \quad i=$ $1,2, \cdots, m$. The coefficients are calculated as $\beta^{*}=\left(F^{T} R^{-1} F\right)^{-1} F^{T} R^{-1} Y$, 
$\gamma^{*}=R^{-1}\left(Y-F \beta^{*}\right)$, where $F$ is the design matrix, written as

$$
F_{m \times p}=\left[f\left(x_{1}\right), f\left(x_{2}\right), \cdots, f\left(x_{m}\right)\right]^{T} .
$$

$m$ is the number of groups of FE model, and in this application, $m=20 . p$ is the number of basis functions, which is determined by the type of basis function. In our experiments, $\mathrm{p}=12$ for first order regression functions. The variable $x_{i}$ is obtained from the FE model by the Latin hypercube sampling method, and $x_{i}$ is normalized to the interval $[0,1]$, as in

$$
\tilde{x}_{i}=\frac{x_{i}-x_{L}}{x_{U}-x_{L}}, \quad \tilde{x}_{i} \in[0,1]
$$

where $\tilde{x}_{i}$ is the normalized data of $x_{i}$ and $x_{L}$ and $x_{U}$ are the minimum and maximum values of the variable $x_{i}$, respectively.

The mean square error of the Kriging prediction model is formulated as (5) [34]

$$
\varphi(x)=\sigma^{2}\left(1+u^{T}\left(F^{T} R^{-1} F\right)^{-1} u-r^{T} R^{-1} r\right),
$$

where $u=F^{T} R^{-1} r-f$ and $\sigma^{2}=\frac{1}{m}\left(Y-F \beta^{*}\right)^{2} R^{-1}\left(Y-F \beta^{*}\right)$ is the maximum likelihood estimate of the variance.

\section{Regression function}

The design matrix $F_{m \times p}$ in the Kriging prediction model is

$$
F_{m \times p}=\left[\begin{array}{ccc}
f_{1}\left(x_{1}\right) & \cdots & f_{p}\left(x_{1}\right) \\
\vdots & \ddots & \vdots \\
f_{1}\left(x_{m}\right) & \cdots & f_{p}\left(x_{m}\right) \\
, & &
\end{array}\right]_{m \times p}
$$

where the basis function $f(x)$ has several forms. Usually, constant and linear forms of the regression function are used to construct the basis function [35].

- Constant form, $p=1$, of which the design matrix $F$ is a column vector.

$$
f_{1}(x)=1 \quad x \in \mathbf{R}^{n}
$$

- Linear form, $p=n+1$, of which the basis function is written as (8).

$$
f_{1}(x)=1, f_{2}(x)=w_{1}, \cdots, f_{p}(x)=w_{n} x \in \mathbf{R}^{n}
$$

where the component form of the estimate point $x$ is expanded as $x=\left[w_{1}, w_{2}, \cdots, w_{n}\right] \in$ $\mathbf{R}^{n}$. In our experiment, both zero order (constant form, $\mathrm{p}=1$ ) and first order (linear form, $\mathrm{p}=12$ ) functions are adopted and compared. 
Correlation function

The Kriging model assumes that the correlation of the output is determined by the distance between the input variables. The correlation function of the input variables is written as the product of $n$ one-dimensional correlation equations, as shown in $(9)$ :

$$
R(w, x)=R(\theta, w, x)=\prod_{j=1}^{n} R_{j}\left(\theta, d_{j}\right)
$$

where $d_{j}=w_{j}-x_{j}$ and $\theta$ is the correlation coefficient. In the Kriging model, the most widely used correlation model is that shown in (10):

$$
R(\theta, w, x)=\prod_{j=1}^{n} \exp \left(-\theta_{j}\left\|d_{j}\right\|^{p}\right)
$$

where $\|\cdot\|$ denotes the Euclidean distance of $d$. When $p=1$ and $p=2$, the exponential and Gaussian correlation functions are as shown in (11) and (12).

$$
\begin{aligned}
& R_{j}\left(\theta, d_{j}\right)=\exp \left(-\theta_{j}\left\|d_{j}\right\|\right) \\
& R_{j}\left(\theta, d_{j}\right)=\exp \left(-\theta_{j}\left\|d_{j}\right\|^{2}\right)
\end{aligned}
$$

The correlation function decreases with the increase in the Euclidean distance $d_{j}$, and a larger correlation coefficient $\theta$ leads to a rapid decline of the correlation function. Substituting the correlation function into (5), the mean square error is a function of the covariance $\sigma^{2}$ and the correlation coefficient $\theta$. The optimal solution to the correlation coefficient $\theta^{*}$ is converted into an unconstrained global optimization problem [36], as shown in (13):

$$
\theta^{*}=\arg \min _{\theta}\left[\psi(\theta) \equiv|R|^{\frac{1}{m}} \sigma^{2}\right]
$$

where $|R|$ is the determinant value of the correlation matrix $R$.

\section{Deformation Modeling of Needle Insertion at a Fixed Depth}

The input and output data are prepared by running the finite element program 20 times, as in [32]. To establish a Kriging model, 19 sets of observation points ( $\left[X_{\text {in }}(1\right.$, : )$\left.\left., \cdots, X_{\text {in }}(i-1,:), X_{\text {in }}(i+1,:), \cdots, X_{\text {in }}(20,:)\right]\right)$ are chosen as the input design sites, and the remaining set $X_{\text {in }}(i,:)$ is used as the test set. The zero order and first order regression functions and the Gaussian and exponential correlation functions are employed to construct the Kriging model for static soft tissue deformation. In this section, the maximum displacements of the marked points are regarded as the static soft tissue deformations. Therefore, there are four kinds of Kriging model, namely, zero order Gaussian, zero order exponential, first order Gaussian and first order exponential, which are shown in Fig. 3. In the figure, the experimental values 

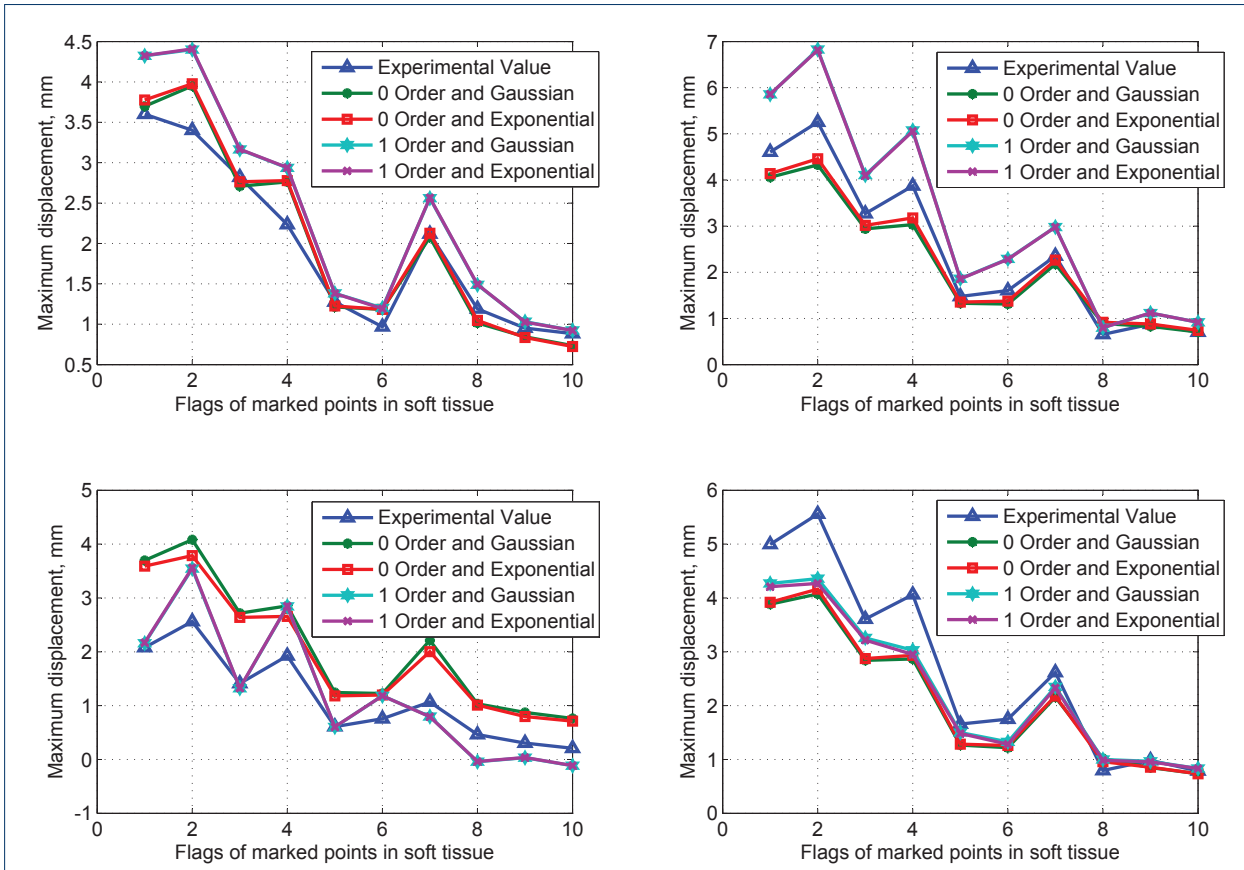

Figure 3: Kriging predictive model for four different combinations

represent the finite element simulation results. The abscissa indicates the number of observation locations, and the ordinate is the value of the maximum resultant displacement. The test set of each run is randomly selected. The upper-left test set is $X_{\text {in }}(5,:)$, the upper-right test set is $X_{\text {in }}(6,:)$, the lower-left test set is $X_{\text {in }}(7,:)$, and the lower-right test set is $X_{\text {in }}(8,:)$. From Fig. 3 , it is also suggested that the closer the tissue is to the needle body, the greater the deformation. To analyze the impacts of the regression function and correlation function on the model, the residual $\mathbf{e}$ of the simulation is defined as the difference between the finite element simulation value $y$ and the Kriging prediction value $\hat{y}$, that is, $\mathbf{e}=\hat{y}-y$. The maximum residual values of the zero order Gaussian, zero order exponential, first order Gaussian and first order exponential Kriging models are $7.5108 \mathrm{~mm}, 7.3327$ $\mathrm{mm}, 3.2752 \mathrm{~mm}$ and $3.2994 \mathrm{~mm}$, respectively. Furthermore, the mean residual of each output response is defined as in (14):

$$
\overline{\mathbf{e}}_{\mathrm{i}}=\frac{1}{\mathrm{~m}} \sum_{j=1}^{\mathrm{m}}\left|\mathbf{e}_{j \mathrm{i}}\right|=\frac{1}{\mathrm{~m}} \sum_{j=1}^{\mathrm{m}}\left|\hat{y}_{j \mathrm{i}}-y_{j \mathrm{i}}\right|,
$$

where $\mathrm{m}$ is the number of finite element program samples, i.e., $m=20$. $\mathrm{i}$ is the position number of the marked positions, $i=1, \cdots, 10$. The mean residuals of the 10 observed locations are shown in Fig. 4. In Fig. 4, the mean residuals of the zero order Gaussian and exponential types are $1.5650 \mathrm{~mm}$ and $1.3992 \mathrm{~mm}$, respectively. The mean maximum residuals of the first order Gaussian and exponential are $0.8941 \mathrm{~mm}$ and $0.9177 \mathrm{~mm}$, respectively. From the prediction results, the first order regression function is better than the zero order regression function, and the Gaussian correlation function is slightly better than the exponential function from 


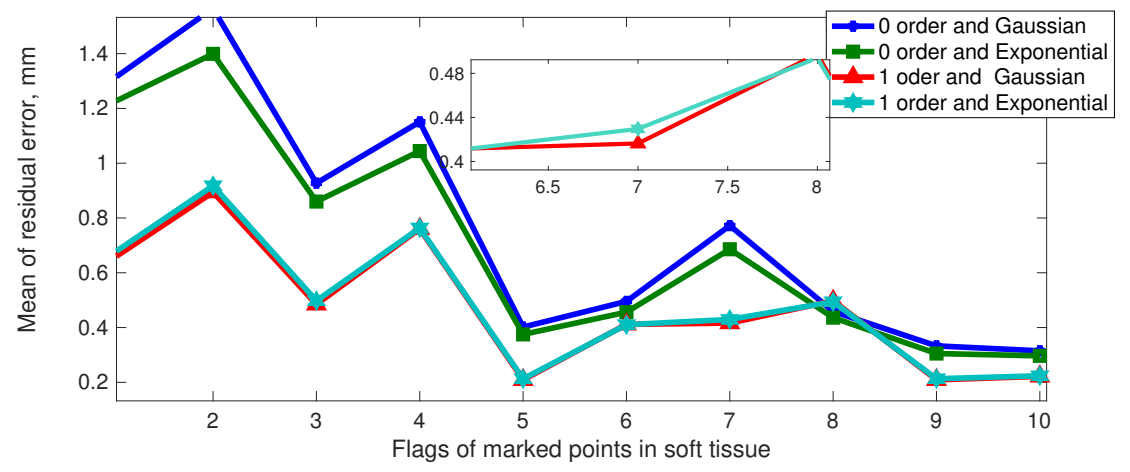

Figure 4: The mean residuals of the Kriging predictions for four different combinations

the enlarged part. The relative mean residual is defined as shown in (15):

$$
\varepsilon=\frac{\overline{\mathbf{e}}_{\mathrm{i}}}{\frac{1}{\mathrm{~m}} \sum_{j=1}^{\mathrm{m}}\left|y_{j \mathrm{i}}\right|} .
$$

\section{Sensitivity analysis of the parameters}

The Kriging-based prediction model of soft tissue deformation takes into account the 11 input parameters in the finite element simulation process, and the effect of the parameters $X=\left[x_{1}, x_{2}, \cdots, x_{11}\right]$ on the tissue deformation $Y=\left[y_{1}, y_{2}, \cdots, y_{10}\right]$ is investigated with the first order Gaussian Kriging-based prediction model in this section.

In this section, the 'local' sensitivity for each variable is investigated, i.e., only one column is varied for each row selected, while the other columns are fixed. Since the 20 groups sample is randomly generated, there is no specific law for the order of rows. Hence, we construct the parameter test matrix by randomly selecting the four rows of data $X(1,:), X(7,:), X(13,:)$ and $X(18,:)$. The varied variable is evenly distributed in the interval $[0,1]$ (the interval is divided into 100 equal parts), while the other input variables retain the same values.

The sensitivity of parameters is reflected by the average sensitivity index (SI), which is the average change in the output response and input variable of the four selected groups of samples, that is, $S I=\frac{1}{4} \sum_{k=1}^{4} \Delta u_{x y}^{k j} / \Delta x_{k i}(j=1, \cdots, 10, i=$ $1, \cdots, 11)$. When the output displacement increases with the variables, the SI is greater than zero. In the opposite case, the SI is less than zero. According to the range of the SI, the 11 input variables can be classified into three types, i.e., leading parameters, nonleading parameters and disturbed parameters, as shown in Fig. 5, where the abscissa represents 11 variables and the ordinate represents the SI. Since there are ten observation points inside the tissue, there are also ten sensitivity indices for each variable; thus, we choose a box plot to show the extremum (black line at both ends), the interquartile range (blue box) and the median (red line) of one group of the dataset. We classify the 11 variables into three categories based on the sensitivity index: The leading parameters include Young's modulus of the soft tissue $x_{1}$, the soft-tissue Poisson ratio $x_{2}$, the needle length $x_{6}$ and the coefficient 


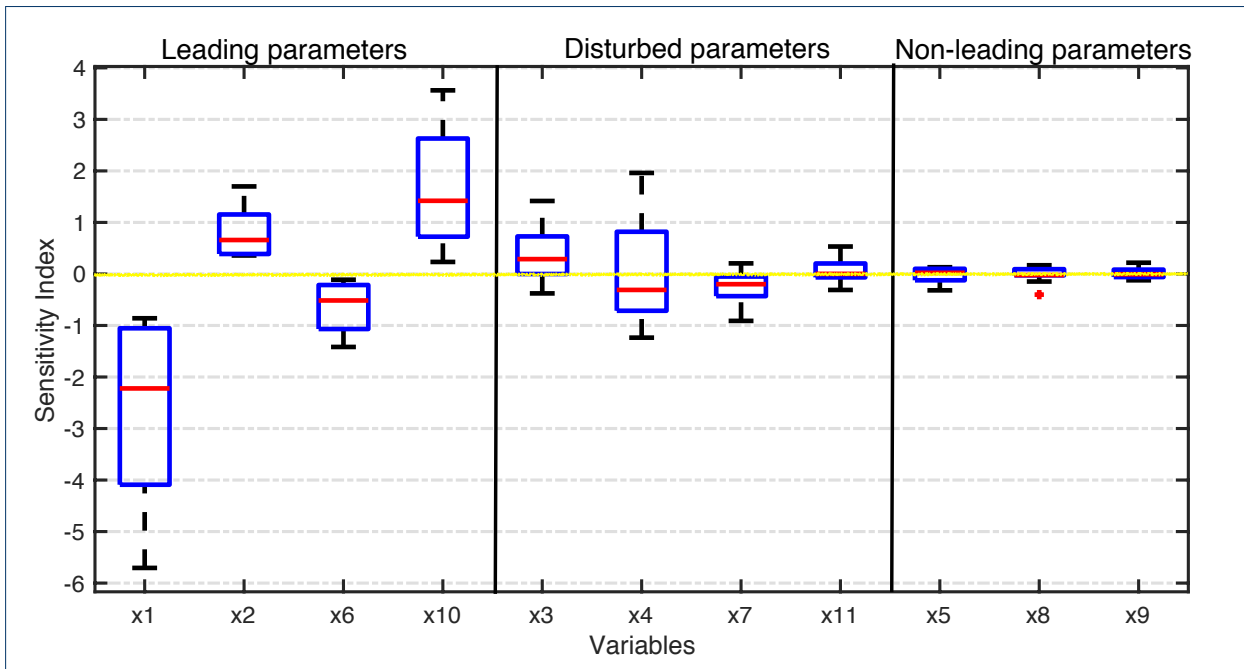

Figure 5: The effects of the leading inputs on the output responses

of friction $x_{10}$, of which the absolute value of the median of the SI is larger than 0.5 and the effect on different markers is consistent (indices are either all distributed above or below zero). For example, the SI of $\mathrm{x} 1$ is always below zero, which means that the soft tissue deformation decreases as $\mathrm{x} 1$ increases. These variables have an obvious impact on the deformation due to the large absolute value of the SI. By contrast, the deformation increases as $\mathrm{x} 2$ increases, with $\mathrm{x} 2$ having a relatively small impact on the deformation. The disturbed parameters include the angle of the needle tip $x_{3}$, insertion angle $x_{4}$, Young's modulus of the needle $x_{7}$ and area density $x_{11}$. The effect of the disturbed parameters varies for different markers. For the disturbed parameters, the sensitivity index covers the yellow line, which means that the deformations of some markers increase with the parameters, while some markers decrease. The nonleading parameters include the needle diameter radius $x_{5}$ and the Rayleigh damping coefficients $\alpha\left(x_{8}\right)$ and $\beta\left(x_{9}\right)$, which means that the absolute value of the median sensitivity is less than 0.02 . The nonleading parameters affect the output displacement slightly and can be regarded as constants.

Sensitivity analysis of the parameters can offer a thorough understanding of the impacts of the input parameters on soft tissue deformation. To ensure rigor of results, other rows of data are also tried in the same way, and the tendencies and slopes came to the same conclusion. The leading and disturbed input parameters play key roles in predicting tissue deformation, directly affecting the overall trend and accuracy of the prediction results. The nonleading parameters have little effect on the prediction results and can be removed from the input parameters if a better real-time performance and lower accuracy are required in the procedure of needle insertion.

The sensitivity analysis provides a basis for reducing the number of input parameters of the Kriging-based prediction model. For a more complex FE model, such as that for a nonlinear, viscoelastic tissue material, the sensitivity analysis of parameters is beneficial for selecting the optimal input parameters and reducing the computation time. 


\section{Deformation Modeling of Needle Insertion at a Series of Depths}

Kriging model of the functional response

According to the continuum mechanics, the motion equation of a point inside the tissue $\mathbf{O}$ in the Euclidean space is expressed as (16):

$$
\mathbf{X}=\chi(\mathbf{x}, t) \mapsto u=\mathbf{X}-\mathbf{x}=\chi(\mathbf{x}, t)-\mathbf{x}
$$

where $\mathbf{x}=x \vec{i}+y \vec{j}+z \vec{k}$ is the position vector at the initial time, $\mathbf{X}=X \vec{i}+Y \vec{j}+Z \vec{k}$ is the position vector at time $\mathrm{t}$, and $u=u_{x} \vec{i}+u_{y} \vec{j}+u_{z} \vec{k}$ is the displacement of point $\mathbf{O}$ at time t. The displacement of tissue nodes is related to the time and space coordination, and it is a functional response with time and space indices. When the Kriging metamodel is used to solve the functional response problem, the calculation of the correlation coefficient of the maximum likelihood estimation includes the inverse matrix and the determinant of the correlation matrix, which leads to the instability and time complexity of the computation [37]. The Kronecker product is employed to construct the correlation matrix and to reduce the computational burden of the Kriging method [38].

The input variables in the $\mathrm{FE}$ simulation experiment are $\mathbf{x}=\left[x_{1}, x_{2}, \cdots, x_{n}\right]^{T}$, $\mathbf{x}_{i} \in \mathbf{R}^{n}, i=1,2, \cdots, m$, and the output response is a function of time $t$. The output response $\mathbf{y}_{i}=\left[y_{i_{1}}, y_{i_{2}}, \cdots, y_{i_{i}}\right]^{T}$ is recorded at each time $\mathbf{t}_{i}=\left[t_{i_{1}}, t_{i_{2}}, \cdots, t_{i_{r_{i}}}\right]^{T}$, where $r_{i}$ denotes the time step. The Kriging model of the functional response is written as (17) on the basis of (2):

$$
y(\mathbf{x}, t)=\mathcal{F}(\mathbf{x}, t) \beta+z(\mathbf{x}, t),
$$

where $y(\mathbf{x}, t)$ is the output response of the input variable $\mathbf{x}$ at time $t . \mathcal{F}(\mathbf{x}, t)=$ $\left[f_{1}(\mathbf{x}, t), \cdots, f_{p}(\mathbf{x}, t)\right]^{T}$ is a set of known polynomial basis functions, where usually $f_{1}(\mathbf{x}, t)=1$. $\beta$ is an unknown basis function coefficient. $z(\mathbf{x}, t)$ is a zero-mean Gaussian random function, the covariance function of which is shown in (18):

$$
\begin{aligned}
V\left(\mathbf{x}_{1}, \mathbf{x}_{2}\right) & =\operatorname{cov}\left(z\left(\mathbf{x}_{1}, t_{1}\right), z\left(\mathbf{x}_{2}, t_{2}\right)\right) \\
& =\sigma^{2} R\left(\mathbf{x}_{1}-\mathbf{x}_{2}, t_{1}-t_{2}\right)
\end{aligned}
$$

Assume that the correlation function $R\left(\mathbf{x}_{1}-\mathbf{x}_{2}, t_{1}-t_{2}\right)$ is the product of $n$ onedimensional correlation equations, as shown in (19):

$$
R\left(\mathbf{x}_{1}-\mathbf{x}_{2}, t_{1}-t_{2}\right)=\left[\prod_{j=1}^{n} R_{i}\left(x_{i 1}-x_{i 2}\right)\right] R_{T}\left(t_{1}-t_{2}\right)
$$

where $R_{i}\left(x_{i 1}-x_{i 2}\right)$ is the correlation function of the $\mathrm{i}^{\text {th }}$ set of variables and $R_{T}\left(t_{1}-\right.$ $\left.t_{2}\right)$ is the correlation function at time $t$.

The functional response is assumed to be the $N \times 1$-dimensional vector $Y=$ $\left[\mathbf{y}_{1}^{T}, \mathbf{y}_{2}^{T}, \cdots, \mathbf{y}_{n}^{T}\right]^{T}$, and $N=\sum_{i=1}^{m} r_{i}$. When the time step is $r$, then $N=m r$. The 
corresponding $N \times n$-dimensional design matrix $X=\left[X_{1}, X_{2}, \cdots, X_{N}\right]$ is represented with the Kronecker notation as shown in (20):

$$
\begin{aligned}
& X_{N \times n}=\left[\mathbf{1}_{r_{1}}^{T} \otimes \mathbf{x}_{1}, \cdots, \mathbf{1}_{r_{n}}^{T} \otimes \mathbf{x}_{n}\right]^{T}
\end{aligned}
$$

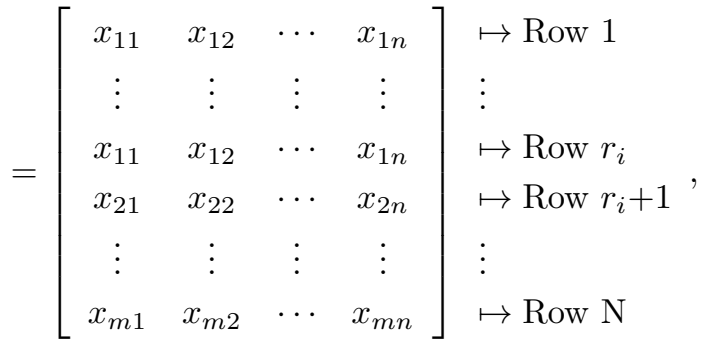

where $\otimes$ is the Kronecker product operator and $\mathbf{1}_{r_{i}}$ is the column vector with length $r_{i}=1$. When the time steps are the same, $X$ is an $m r \times n$ matrix. $T=$ $\left[\mathbf{t}_{1}^{T}, \cdots, \mathbf{t}_{n}^{T}\right]^{T}=\left[t_{1}^{*}, t_{2}^{*}, \cdots, t_{N}^{*}\right]^{T} \in \mathbf{R}^{N \times 1}$ is the corresponding functional space. Combined with equations (2) and (17), the Kriging model of the functional response is written as shown in (21):

$$
\hat{y}(\mathbf{x}, t)=\mathcal{F}(\mathbf{x}, t) \hat{\beta}+R^{T}(\mathbf{x}, t) \mathcal{R}_{X, \mathbf{t}}^{-1}(Y-\mathbf{F} \hat{\beta}),
$$

where $\mathcal{F}$ is written as

$$
\begin{aligned}
& \mathbf{F}=\left[\mathcal{F}\left(X_{1}, t_{1}^{*}\right), \cdots, \mathcal{F}\left(X_{N}, t_{N}^{*}\right)\right]^{T} \\
& \hat{\beta}=\left(\mathbf{F}^{T} \mathcal{R}_{X, \mathbf{t}}^{-1} \mathbf{F}\right)^{-1} \mathbf{F}^{T} \mathcal{R}_{X, \mathbf{t}}^{-1} Y \\
& R(\mathbf{x}, t)=\left[R\left(\mathbf{x}-X_{1}, t-t_{1}^{*}\right), \cdots, R\left(\mathbf{x}-X_{N}, t-t_{N}^{*}\right)\right]^{T}
\end{aligned}
$$

in which $\mathcal{R}_{X, \mathbf{t}}$ is an $N \times N$ correlation matrix, the elements of which are $R\left(X_{i}-\right.$ $\left.X_{j}, t_{i}^{*}-t_{j}^{*}\right)$. The correlation coefficient $\theta$ is optimized with (13). The optimization of the correlation coefficient requires a large number of solutions of $\mathcal{R}_{X, \mathbf{t}}^{-1}$ and $\left|\mathcal{R}_{X, \mathbf{t}}\right|$ so that the computational cost is extremely high.

In this paper, we consider the functional output on the regular grid, i.e., $\mathbf{t}_{1}=\cdots=$ $\mathbf{t}_{n}=t, r_{1}=\cdots=r_{n}=r$. The correlation matrix $\mathcal{R}_{X, \mathbf{t}}$ of the functional response is written as (23):

$$
\mathcal{R}_{X, \mathbf{t}}=\mathcal{R}_{X} \otimes \mathcal{R}_{\mathbf{t}}
$$

where $\mathcal{R}_{X}$ is an $m \times m$-dimensional correlation matrix whose elements are $R\left(\mathbf{x}_{1}-\mathbf{x}_{2}\right)$ and $\mathcal{R}_{t}$ is an $r \times r$-dimensional correlation matrix whose elements are $R_{T}\left(t_{i}-t_{j}\right)$. The Gaussian correlation function is used to construct the correlation matrix, and the inverse of the correlation matrix is simplified to $\mathcal{R}_{X, \mathbf{t}}^{-1}=\mathcal{R}_{X}^{-1} \otimes \mathcal{R}_{\mathbf{t}}{ }^{-1}$. Substituting this matrix into (21), we can write the Kriging model of the functional response as (24):

$$
\hat{y}(\mathbf{x}, t)=\mathcal{F}(\mathbf{x}, t) \hat{\beta}+R^{T}(\mathbf{x}, t) \mathcal{R}_{X}^{-1} \otimes \mathcal{R}_{\mathbf{t}}{ }^{-1}(Y-\mathbf{F} \hat{\beta}) .
$$

219 The computational complexity of the inversion matrix is reduced from $O\left(n^{3} m^{3}\right)$ to $O\left(n^{3}+m^{3}\right)$ with the Kronecker product operator. 
(17) takes account of the coupling effect between the input variable $\mathbf{x}$ and time $t$. The basis function $\mathcal{F}(\mathbf{x}, t)$ is identified by estimating the average value of the functional response. In the case of $r_{1}=\cdots=r_{n}=r$, we define $\bar{e}_{\cdot j}=\frac{1}{m} \sum_{i=1}^{m}\left(y_{i j}-\overline{y_{i}}\right.$. $)$ and $\bar{y}_{i \cdot}=\frac{1}{r} \sum_{l=1}^{r} y_{i l} \cdot \bar{e}=\left[\bar{e}_{\cdot 1}, \cdots, \bar{e}_{\cdot r}\right]^{T}$ and $\bar{y}=\left[\bar{y}_{1}, \cdots, \bar{y}_{m}.\right]$ are fitted as shown in (25).

$$
\begin{aligned}
\bar{e}(t) & =k^{T}(t) \beta_{t}+z(t) \\
\bar{y}(\mathbf{x}) & =g^{T}(\mathbf{x}) \beta_{x}+z(\mathbf{x})
\end{aligned}
$$

The unknown polynomial basis functions $k(x)$ and $g(x)$ are constructed using the regression function. Furthermore, the basis function in (24) is written as $\mathcal{F}(\mathbf{x}, t)=\left[1, k^{T}(x), g^{T}(x)\right]^{T}$, and the matrix $\mathbf{F}$ is expressed as $\mathbf{F}=$ $\left[\mathcal{F}\left(X_{1}, t_{1}^{*}\right), \cdots, \mathbf{F}\left(X_{N}, t_{N}^{*}\right)\right]^{T}$.

\section{Deformation Modeling of Needle Insertion at a Series of Depths}

One hundred sets of input variables are generated with LHS to construct the functional response Kriging model. After the normalized inverse operation, the finite element program runs 100 times to generate the deformations in the $X$ and $Y$ directions at 10 marked points for 31 time steps, as shown in Fig. 2. For needle insertion at a constant speed, the insertion time is replaced with the depth $d$. Here, the 31 time steps are expressed as $d=[1.0 \mathrm{~mm}, 2.0 \mathrm{~mm}, 5.0 \mathrm{~mm}, \cdots, 89 \mathrm{~mm}]$, and the increment of the insertion depth is $3 \mathrm{~mm}$, except in the first step. Forty sets of data are randomly selected from 100 sets of records and used to construct the functional Kriging prediction model of soft tissue deformation, and an independent set of data is used to test the model. According to the former analysis, the polynomial basis functions are first order regression functions, and the correlation functions are Gaussian random functions. To evaluate the real-time performance of the Kriging module, the mean residual $\overline{\tilde{e}}_{i}$ and the relative mean residual $\overline{\tilde{\varepsilon}}_{i}$ at each time step (insertion depth) are defined for each observation location, written as (26):

$$
\begin{aligned}
& \overline{\tilde{\mathbf{e}}}_{\mathrm{i}}=\frac{1}{\mathrm{r}} \sum_{j=1}^{\mathrm{r}}\left|\mathbf{e}_{j \mathrm{i}}\right|=\frac{1}{\mathrm{r}} \sum_{j=1}^{\mathrm{r}}\left|\hat{y}_{j \mathrm{i}}-y_{j \mathrm{i}}\right| \\
& \overline{\tilde{\varepsilon}}_{\mathrm{i}}=\frac{1}{\mathrm{r}} \sum_{j=1}^{\mathrm{r}} \frac{\left|\hat{y}_{j \mathrm{i}}-y_{j \mathrm{i}}\right|}{\left|y_{j \mathrm{i}}\right|},
\end{aligned},
$$

where $\mathrm{r}$ is the total number of puncturing steps, i.e., $\mathrm{r}=31$, and $\mathrm{i}$ is the position number of the output response, where $\mathrm{i}=1, \cdots, 10$.

The procedure of needle insertion is recorded from the time of contact between the soft tissue and the needle tip to the time at which a depth of $90 \mathrm{~mm}$ is reached inside the tissue. With the functional response Kriging model, the displacement in the $X$ and $Y$ directions can be predicted, and Fig. 6 plots the displacements of $N 2$ shown in Fig. 2, which is close to the needle. In Fig. 6, 'Kriging value' denotes the prediction results obtained using the functional response Kriging model, and 'FEM' denotes the results obtained with a finite element simulation. From Fig. 6, the displacement fluctuations at the initial and final time steps are relatively large, while the fluctuations between these two time steps are smaller. The functional 

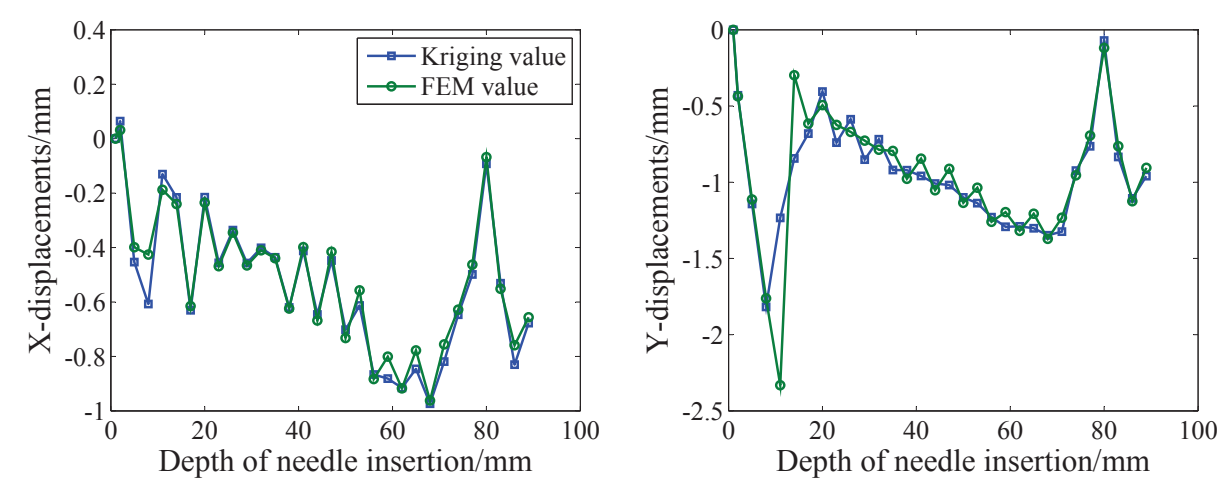

Figure 6: Kriging predictions for insertion depth-tissue displacement curve (N2) away from the needle.
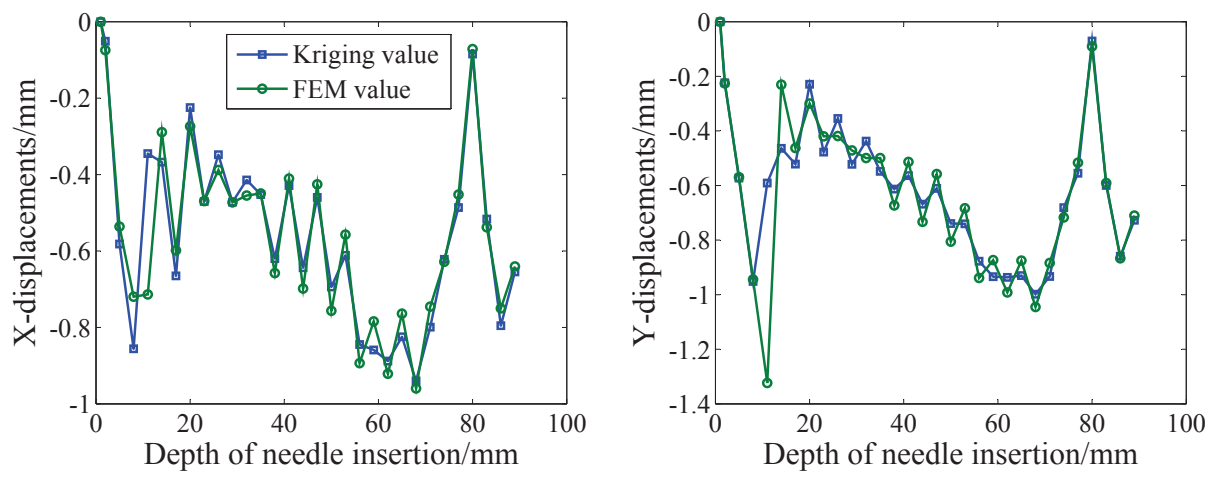

Figure 7: Kriging predictions for insertion depth-tissue displacement curve (N8)

response Kriging model follows the law of tissue deformation during the needle insertion procedure. The model can also predict the displacements at other locations at different time steps very well. Fig. 7 shows the displacements at $N 8$, which is far

With (26), the mean residuals and relative mean residuals of the 10 locations are as listed in Table 2. Overall, the Kriging model smooths the original finite element

Table 2: Kriging prediction errors at different positions

\begin{tabular}{ccccccccccc}
\hline Error & $\mathrm{N} 1$ & $\mathrm{~N} 2$ & $\mathrm{~N} 3$ & $\mathrm{~N} 4$ & $\mathrm{~N} 5$ & $\mathrm{~N} 6$ & $\mathrm{~N} 7$ & $\mathrm{~N} 8$ & $\mathrm{~N} 9$ & $\mathrm{~N} 10$ \\
\hline$X_{\max }$ & 0.19 & 0.18 & 0.13 & 0.13 & 0.09 & 0.09 & 0.37 & 0.37 & 0.23 & 0.31 \\
$Y_{\max }$ & 1.24 & 1.10 & 0.84 & 0.76 & 0.56 & 0.52 & 1.06 & 0.73 & 0.55 & 0.52 \\
$X_{\operatorname{mean}}$ & 0.04 & 0.03 & 0.03 & 0.03 & 0.02 & 0.02 & 0.05 & 0.05 & 0.03 & 0.04 \\
$Y_{\text {mean }}$ & 0.12 & 0.11 & 0.08 & 0.08 & 0.07 & 0.06 & 0.10 & 0.07 & 0.05 & 0.05 \\
$X_{\text {re }}$ & $12 \%$ & $12 \%$ & $13 \%$ & $13 \%$ & $12 \%$ & $12 \%$ & $10 \%$ & $10 \%$ & $10 \%$ & $10 \%$ \\
$Y_{\text {re }}$ & $14 \%$ & $16 \%$ & $14 \%$ & $16 \%$ & $15 \%$ & $16 \%$ & $15 \%$ & $13 \%$ & $13 \%$ & $12 \%$ \\
\hline${ }^{*} \cdot \max$ maximum residual. ${ }^{*} \cdot$ mean & mean residual. ${ }^{*} \cdot{ }_{\text {re }}$ relative mean residual. &
\end{tabular}

model, and the prediction accuracy of the initial stage is poor. The maximum residuals of the Kriging predictions and finite element calculations in the $X$ and $Y$ directions are $0.37 \mathrm{~mm}$ and $1.24 \mathrm{~mm}$, respectively, and both occur in the fifth 


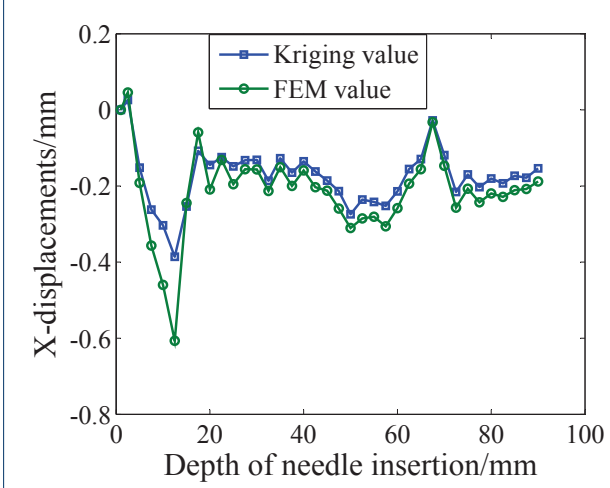
the FEM model.

time step, at which the needle tip punctures the surface of the soft tissue. The average residuals in the $X$ and $Y$ directions occur within $0.05 \mathrm{~mm}$ and $0.12 \mathrm{~mm}$, respectively, and the relative mean residual is at most $16 \%$.

The runtime is also compared. Both the Kriging model and FEM model run on the same computer with macOS Mojave 10.14.6, a $2.7 \mathrm{~Hz}$ Intel Core i5 and 8 GB of RAM, and the program is written in MATLAB 2019b. The average runtime of 5 runs is recorded, with the average time for the Kriging-based method being 0.0294 $\mathrm{s}$ and that of the FEM model being $4.6912 \mathrm{~s}$. Note that neither of the two reported times includes a visualization of the soft tissue deformation process. The reported results indicate that the Kriging model runs approximately 160 times faster than

\section{Adaptation of the Deformation Modeling to Step Variations}

From the above analysis, the Kriging prediction model based on the observations at 31 time steps can reflect the soft tissue deformation at fixed (known) time steps. However, it is necessary to obtain the motion information of the sensitive area inside the soft tissue at any time step for both the path planning and the virtual training system. In this section, the adaptation to different time steps is studied. Additional insertion time steps are expressed as $d_{1}=[1.0 \mathrm{~mm}, 2.5 \mathrm{~mm}, 5.0 \mathrm{~mm}, \cdots, 90 \mathrm{~mm}]$, the insertion depth increment is $2.5 \mathrm{~mm}$ and the total depth achieved by the needle is $90 \mathrm{~mm}$. The displacements of 10 observation locations at $d_{1}$ insertion time steps are recorded to compare with the Kriging model. Forty sets of data randomly selected from 100 sets of records are used to construct the real-time Kriging prediction model of soft tissue deformation, and an independent set of data is used to test the model. Fig. 8 shows a comparison between the prediction and the computer-based experimental results at the $N 3$ location. From Fig. 8, the Kriging model with a

Figure 8: Kriging predictions for insertion depth-tissue displacement curve (N3)

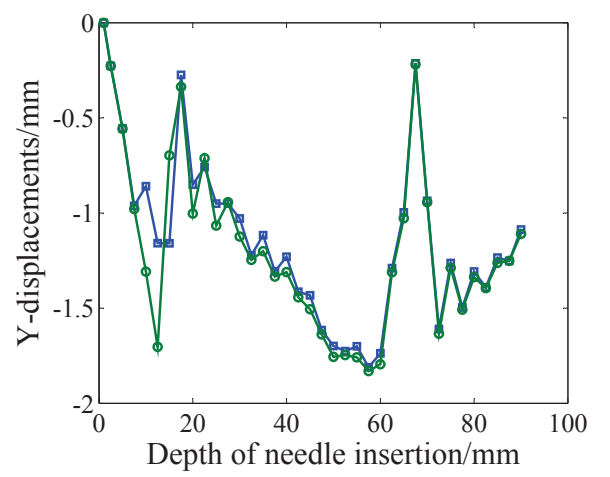

functional response can fit the computer-based experimental results very well and can reflect the fluctuation trends at the initial and end time steps. The maximum residual occurs at the 6 th insertion time step, and its maximum residuals and relative mean residuals in the $X$ direction are $0.22 \mathrm{~mm}$ and $21 \%$, respectively. The maximum residuals and relative residuals in the $Y$ direction are $0.55 \mathrm{~mm}$ and $8 \%$, respectively. The maximum residuals and relative mean residuals of the remaining 
Table 3: Kriging prediction errors at different positions

\begin{tabular}{cccccccccc}
\hline Error & $\mathrm{N} 1$ & $\mathrm{~N} 2$ & $\mathrm{~N} 4$ & $\mathrm{~N} 5$ & $\mathrm{~N} 6$ & $\mathrm{~N} 7$ & $\mathrm{~N} 8$ & $\mathrm{~N} 9$ & $\mathrm{~N} 10$ \\
\hline$X_{\max }$ & 0.31 & 0.20 & 0.22 & 0.16 & 0.11 & 0.31 & 0.29 & 0.24 & 0.28 \\
$Y_{\max }$ & 0.76 & 0.92 & 0.55 & 0.37 & 0.37 & 0.51 & 0.37 & 0.32 & 0.29 \\
$X_{\text {re }}$ & $24 \%$ & $33 \%$ & $20 \%$ & $21 \%$ & $49 \%$ & $25 \%$ & $25 \%$ & $15 \%$ & $12 \%$ \\
$Y_{\text {re }}$ & $6 \%$ & $7 \%$ & $7 \%$ & $8 \%$ & $7 \%$ & $6 \%$ & $6 \%$ & $6 \%$ & $6 \%$ \\
\hline${ }^{*} \cdot \max$ maximum residual, ${ }^{*} \cdot$ re relative mean residual.
\end{tabular}

nine observation positions are listed in Table 3. From Table 3, the maximum residual in the $X$ direction occurs at $N 1$ and $N 7$; its value is $0.31 \mathrm{~mm}$. The maximum residual in the $Y$ direction appears at $\mathrm{N} 2$; its value is $0.92 \mathrm{~mm}$. The relative mean residual in the $Y$ direction is less than $8 \%$ and has a relatively high accuracy. For the real insertion procedure, the displacements in the $X$ direction are very small, sometimes approaching zero; hence, the relative mean residual is larger than $20 \%$. However, the absolute mean residual of each location is less than $0.31 \mathrm{~mm}$, which can meet the estimation accuracy.

\section{Conclusions and Future Work}

In this paper, a computer-based experimental analysis of the Kriging metamodel is presented to predict the deformations of soft tissues. The input data include the material properties of the tissues and needle, the geometrical properties of the needle, and the solver parameters, and they are sampled by the LHS method. The corresponding output dataset is generated by an accurate needle-tissue coupling FE model offline. Ten markers are used to represent the deformations at different positions inside the tissues. Unlike other mechanical-based simulation models, our model can consider both the accuracy and efficiency if high-precision data of the original dataset are well prepared. The results suggest that the proposed Krigingbased model with first order regression and Gaussian correlation functions can well reflect the mechanism of soft tissue deformation. The functional response Krigingbased model can provide feedback regarding deformation at a series of depths for the SPS, of which the time and space indices are both taken into account. The reported time indicates that the Kriging model runs approximately 160 times faster than the original FEM model. Moreover, we also verify that the model has excellent adaptation to step variations. Compared with the ground truth, the maximum residual is less than $0.92 \mathrm{~mm}$, which can satisfy the requirements of the SPS.

The performance of the proposed Kriging-based model depends on the accuracy of the dataset generated by the mechanical-based simulation greatly. Therefore, future work will focus on combinations with a more accurate simulation model, considering complex tissue behavior ranging from hyperelasticity to viscoelasticity, and the needle deflection will be modeled at the same time. The sensitivity of additional parameters will be analyzed to improve the accuracy and efficiency of the Kriging-based model.

\section{Declarations}

\section{Availability of data and materials}

The datasets analysed during the current study are available from the corresponding author on reasonable request. 


\section{Competing interests}

The authors declare that they have no competing interests

\section{Funding}

This work was supported in part by the National Natural Science Foundation of China under grant No. 81827804 and 51665049 and by the Zhejiang Provincial Natural Science Foundation of China under grant No. LSD19H180004.

\section{Authors' contributions}

YL discovered the Kriging based tissue deformation online prediction, ML designed and conducted the sensitivity experiments to find out the effects of different factors and ML assisted with improving the quality of the manuscript. DG interpreted the experimental results. All authors read and approved the final manuscript.

\section{Acknowledgements}

No applicable

\section{Authors' information}

YL received a Ph.D. in Mechanical Engineering from the University of Michigan, Ann Arbor, in 2007. He is currently a professor at the State Key Laboratory of Fluid Power Transmission and Control, Department of Mechanical Engineering, Zhejiang University, Hangzhou. His major research interests include fault diagnosis and maintenance in networked systems, modeling and human-machine interface design for minimally invasive surgery; ML is currently pursuing a $\mathrm{Ph} . \mathrm{D}$. at the School of Mechanical Engineering, Zhejiang University, Hangzhou, China. Her current research interests include surgical simulation and robotics; DG received a Ph.D. in Mechatronics Engineering from Zhejiang University, Hangzhou, in 2017 and an M.S. in Mechatronics Engineering from Tsinghua University, Beijing, in 2007. He is currently a professor at the School of Mechanical Engineering, Qinghai University, Xining. His major research interests include computer-aided medical engineering and smart maintenance and operation for a photovoltaic system.

Author details

${ }^{1}$ Department of Mechanical Engineering, Zhejiang University, Zheda Road No.38, Hangzhou, 310027 China

${ }^{2}$ School of Mechanical Engineering Qinghai University, Xining, 810016 China.

References

1. Abolhassani, N., Patel, R.V., Moallem, M.: Needle insertion into soft tissue:a survey. Medical Engineering Physics 29, 413-431 (2007)

2. Gao, D., Lei, Y., Zheng, H.: Needle steering for robot-assisted insertion into soft tissue: a survey. Chinese Journal of Mechanical Engineering 25, 629-638 (2012)

3. O'Flynn, E., Wilson, A., Michell, M.: Image-guided breast biopsy: state-of-the-art. Clinical radiology 65(4), 259-270 (2010)

4. Goksel, O., Sapchuk, K., Salcudean, S.E.: Haptic simulator for prostate brachytherapy with simulated needle and probe interaction. Haptics, IEEE Transactions on 4(3), 188-198 (2011)

5. Cowan, N.J., Goldberg, K., Chirikjian, G.S., Fichtinger, G., Alterovitz, R., Reed, K.B., Kallem, V., Park, W., Misra, S., Okamura, A.M.: Robotic needle steering: Design, modeling, planning, and image guidance. In: Surgical Robotics, pp. 557-582. Springer, New York (2011)

6. Weichao, S., Huijun, G., Okyay, K.: Finite frequency control for vehicle active suspension systems. IEEE Transactions on Control Systems Technology 19(2), 416-422 (2011)

7. Okamura, A.M., Simone, C., O'Leary, M.D.: Force modeling for needle insertion into soft tissue. IEEE Transactions on Biomedical Engineering 51, 707-716 (2004)

8. Wan, G., Wei, Z., Gardi, L.: Brachytherapy needle deflection evaluation and correction. Medical Physics 32, 902-909 (2005)

9. Abolhassani, N., Patel, R.V., Ayazi, F.: Minimization of needle deflection in robot-assisted percutaneous therapy. The International Journal of Medical Robotics and Computer Assisted Surgery 3, 140-148 (2007) 
10. Goksel, O., Dehghan, E., Salcudean, S.E.: Modeling and simulation of flexible needles. Medical Engineering Physics 31, 1069-1078 (2009)

11. Misra, S., Reed, K.B., Schafer, B.W.: Observations and Models for Needle-tissue Interactions, pp. $2687-2692$. Proceedings of 2009 IEEE ICRA, Kobe, Japan (2009)

12. Hing, J.T., Brooks, A.D., Desai, J.P.: Reality-based needle insertion simulation for haptic feedback in prostate brachytherapy. In: Proceedings of the 2006 IEEE International Conference on Robotics and Automation, pp. 619-624 (2006). IEEE

13. DiMaio, S.P., Salcudean, S.E.: Needle insertion modeling and simulation. Robotics and Automation, IEEE Transactions on 19(5), 864-875 (2003)

14. Misra, S., Ramesh, K.T., Okamura, A.M.: Modeling of tool-tissue interactions for computer-based surgical simulation: A literature review. Presence 17(5), 463-491 (2014)

15. Kobayashi, Y., Onishi, A., Hoshi, T., Kawamura, K., Hashizume, M., Fujie, M.G.: Development and validation of a viscoelastic and nonlinear liver model for needle insertion. International journal of computer assisted radiology and surgery $4(1), 53-63$ (2009)

16. Misra, S., Macura, K.J., Ramesh, K.T., Okamura, A.M.: The importance of organ geometry and boundary constrains for planning of medical interventions. Medical Engineering Physics 31, 195-206 (2009)

17. Buijs, J.o.d., Hansen, H.H.G., Lopata, R.G.P., Korte, C.L.d., Misra, S.: Predicting target displacements using ultrasound elastography and finite element modeling. IEEE Transactions on Biomedical Engineering 58(11), 3143-3155 (2011)

18. Takabi, B., Tai, B.L.: A review of cutting mechanics and modeling techniques for biological materials. Medical Engineering \& Physics 45, 1 (2017)

19. Gokgol, C., Basdogan, C., Canadinc, D.: Estimation of fracture toughness of liver tissue: experiments and validation. Medical Engineering \& Physics 34(7), 882-891 (2012)

20. Singh, S., Lo, M.C., Damodaran, V.B., Kaplan, H.M., Kohn, J., Zahn, J.D., Shreiber, D.I.: Modeling the insertion mechanics of flexible neural probes coated with sacrificial polymers for optimizing probe design. Sensors 16(3), 330 (2016)

21. Oldfield, M., Dini, D., Giordano, G., Rodriguez, Y.B.F.: Detailed finite element modelling of deep needle insertions into a soft tissue phantom using a cohesive approach. Computer Methods in Biomechanics \& Biomedical Engineering 16(5), 530-543 (2013)

22. Paulus, C.J., Suwelack, S., Schoch, N., Speidel, S., Dillmann, R., Heuveline, V.: Simulation of complex cuts in soft tissue with the extended finite element method (x-fem). Preprint (02) (2014)

23. Cheng, Q.Q., Liu, P.X., Lai, P.H., Zou, Y.N.: An interactive meshless cutting model for nonlinear viscoelastic soft tissue in surgical simulators. IEEE Access (2017)

24. Zou, Y., Xu, S., Lai, P., Liu, P.X., Cheng, Q.: A novel haptic interactive approach to simulation of surgery cutting based on mesh and meshless models. Journal of Healthcare Engineering,2018,(2018-4-15) 2018(5), 1-16 (2018)

25. Yuan, M., Chen, Z., Yao, B., Zhu, X.: Time optimal contouring control of industrial biaxial gantry: A high-efficient analytical solution of trajectory planning. IEEE/ASME Transactions on Mechatronics (2016). doi:10.1109/TMECH.2016.2592518

26. Chen, Z., Pan, Y.-J., Gu, J.: Integrated adaptive robust control for multilateral teleoperation systems under arbitrary time delays. International Journal of Robust and Nonlinear Control 26(12), 2708-2728 (2016)

27. Kleijnen, J.P.: Kriging metamodeling in simulation: A review. European Journal of Operational Research 192(3), 707-716 (2009)

28. Meckesheimer, M., Booker, A.J., Barton, R.R., Simpson, T.W.: Computationally inexpensive metamodel assessment strategies. AIAA journal 40(10), 2053-2060 (2002)

29. Hung, Y., Joseph, V.R., Melkote, S.N.: Analysis of computer experiments with functional response. Technometrics 57(1), 35-44 (2015)

30. Yao, J., Jiao, Z., Ma, D., Yan, L.: High-accuracy tracking control of hydraulic rotary actuators with modeling uncertainties. IEEE/ASME Transactions on Mechatronics 19(2), 633-641 (2014)

31. Yao, J., Jiao, Z., Ma, D.: Extended-state-observer-based output feedback nonlinear robust control of hydraulic systems with backstepping. IEEE Transactions on Industrial Electronics 61(61), 6285-6293 (2014)

32. Gao, D., Lei, Y., Lian, B., Yao, B.: Modeling and simulation of flexible needle insertion into soft tissue using modified local constraints. Journal of Manufacturing Science and Engineering 138(12), 121012 (2016)

33. Hung, Y., Joseph, V.R., Melkote, S.N.: Analysis of computer experiments with functional response. Technometrics 57(1), 35-44 (2015)

34. Kleijnen, J.P.: Kriging metamodeling in simulation: A review. European Journal of Operational Research 192(3), 707-716 (2009)

35. Lophaven, S.N., B, N.H., J, S.: Dace - a matlab kriging toolbox - version 2.0, 2002-12 (2002)

36. Sacks, J., Welch, W.J., Mitchell, T.J., Wynn, H.P.: Design and analysis of computer experiments. Statistical science, 409-423 (1989)

37. Joseph, V.R., Kang, L.: Regression-based inverse distance weighting with applications to computer experiments. Technometrics 53(3), 254-265 (2011)

38. Williams, B., Higdon, D., Gattiker, J., Moore, L., McKay, M., Keller-McNulty, S., et al.: Combining experimental data and computer simulations, with an application to flyer plate experiments. Bayesian Analysis 1(4), 765-792 (2006) 
Figures

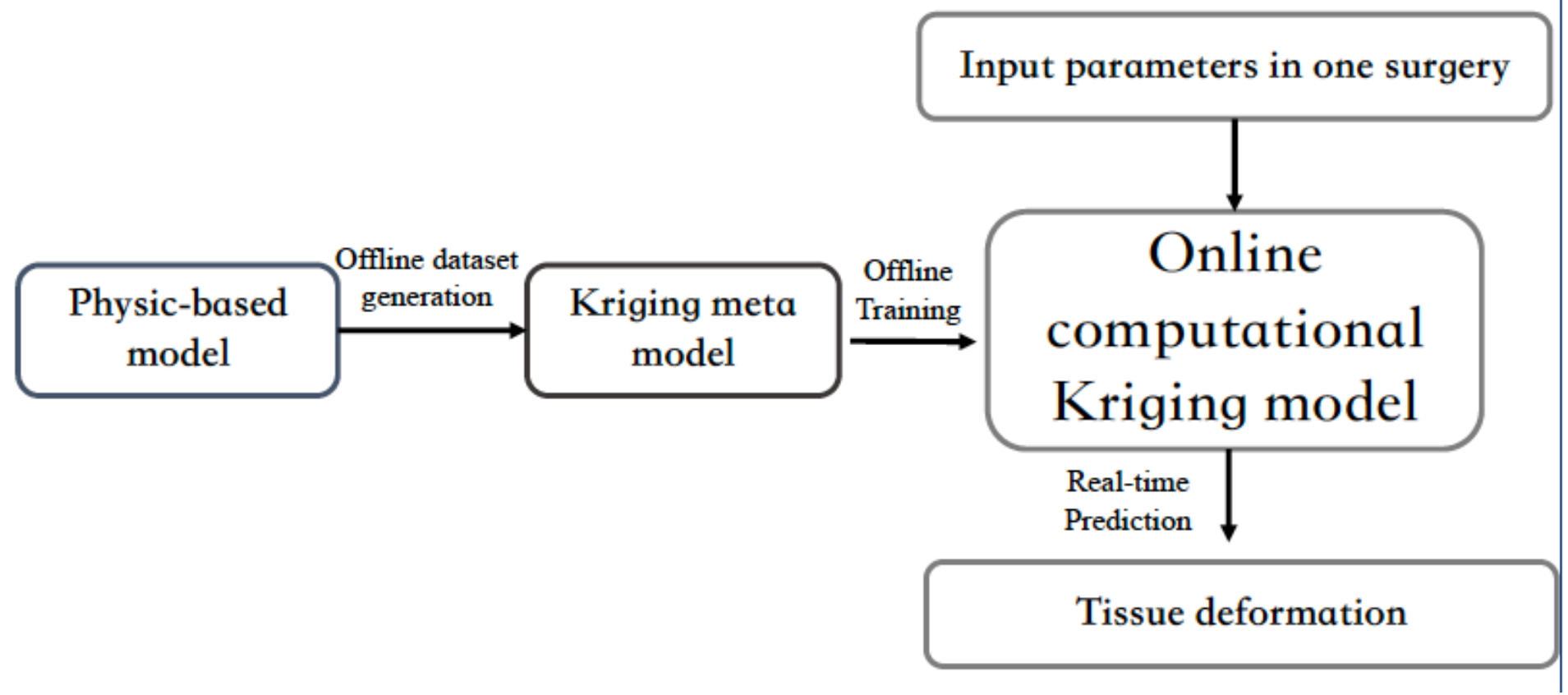

Figure 1

Flowchart of Kriging online prediction 


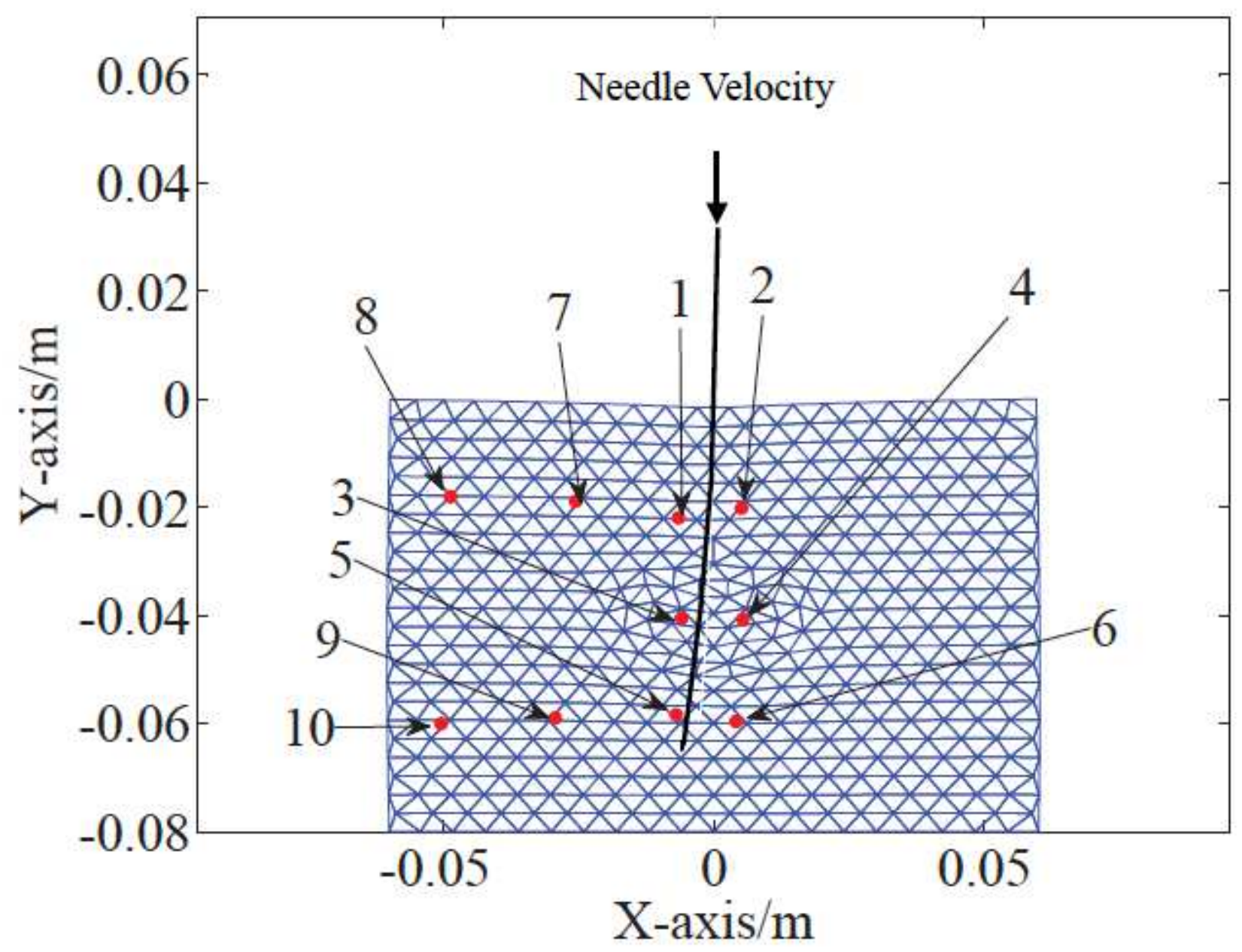

Figure 2

10 observation points in the needle insertion procedure for the Kriging-based model 

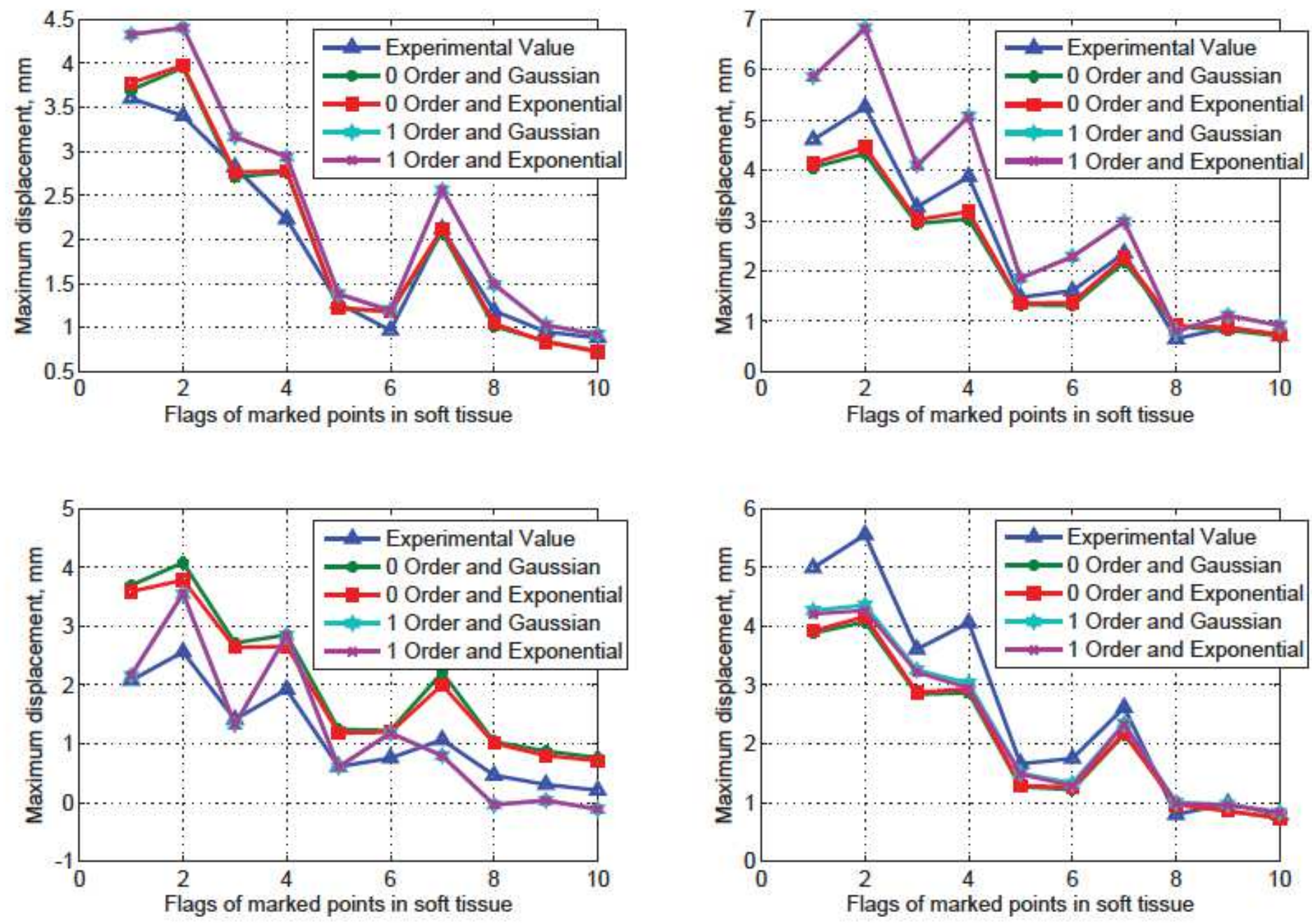

Figure 3

Kriging predictive model for four different combinations

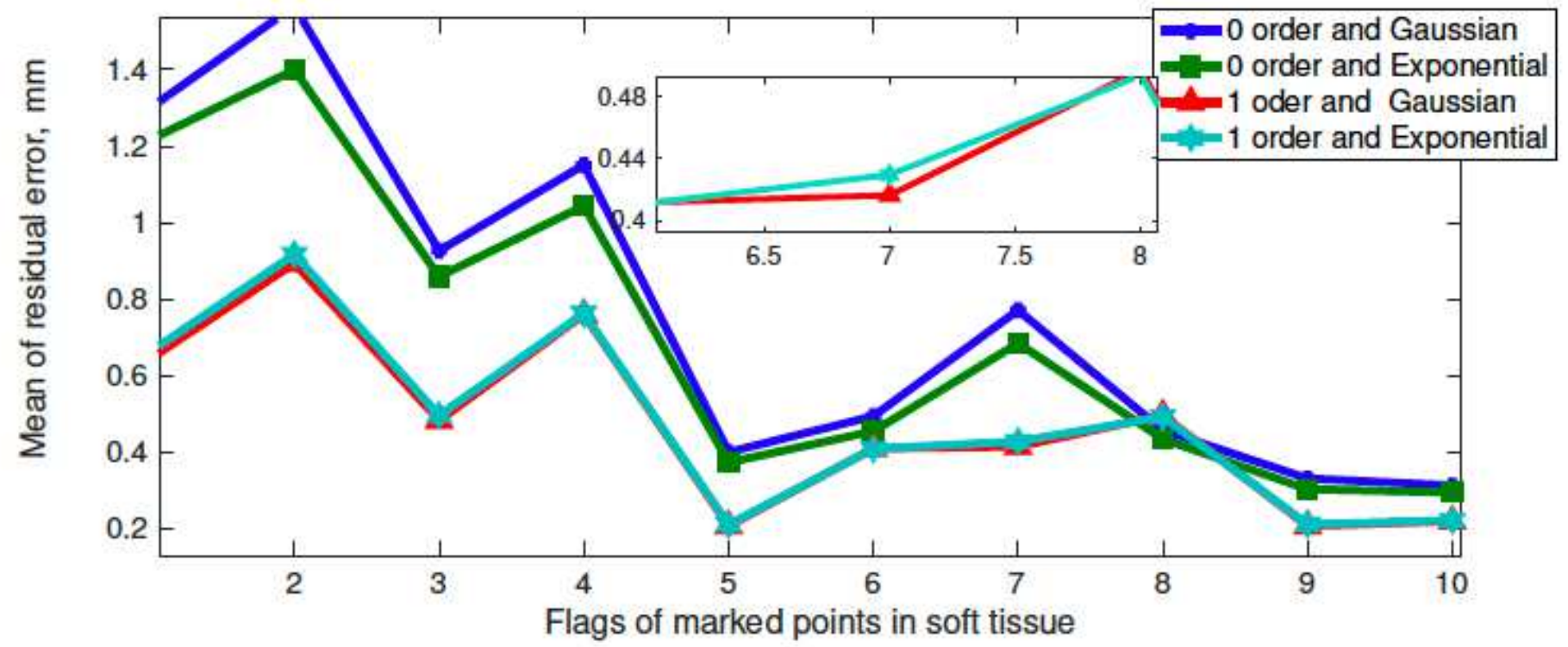


Figure 4

The mean residuals of the Kriging predictions for four different combinations

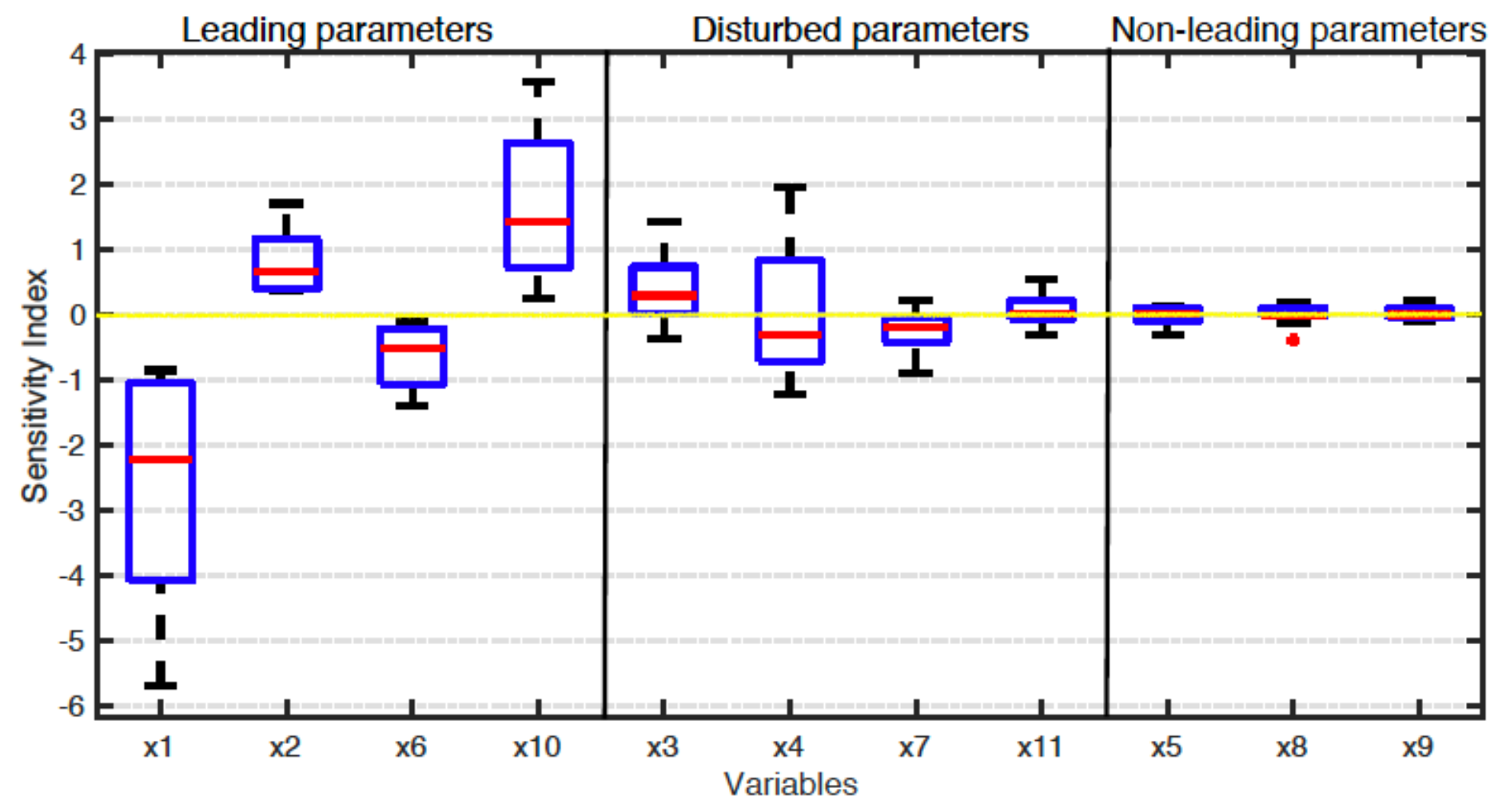

Figure 5

The effects of the leading inputs on the output responses
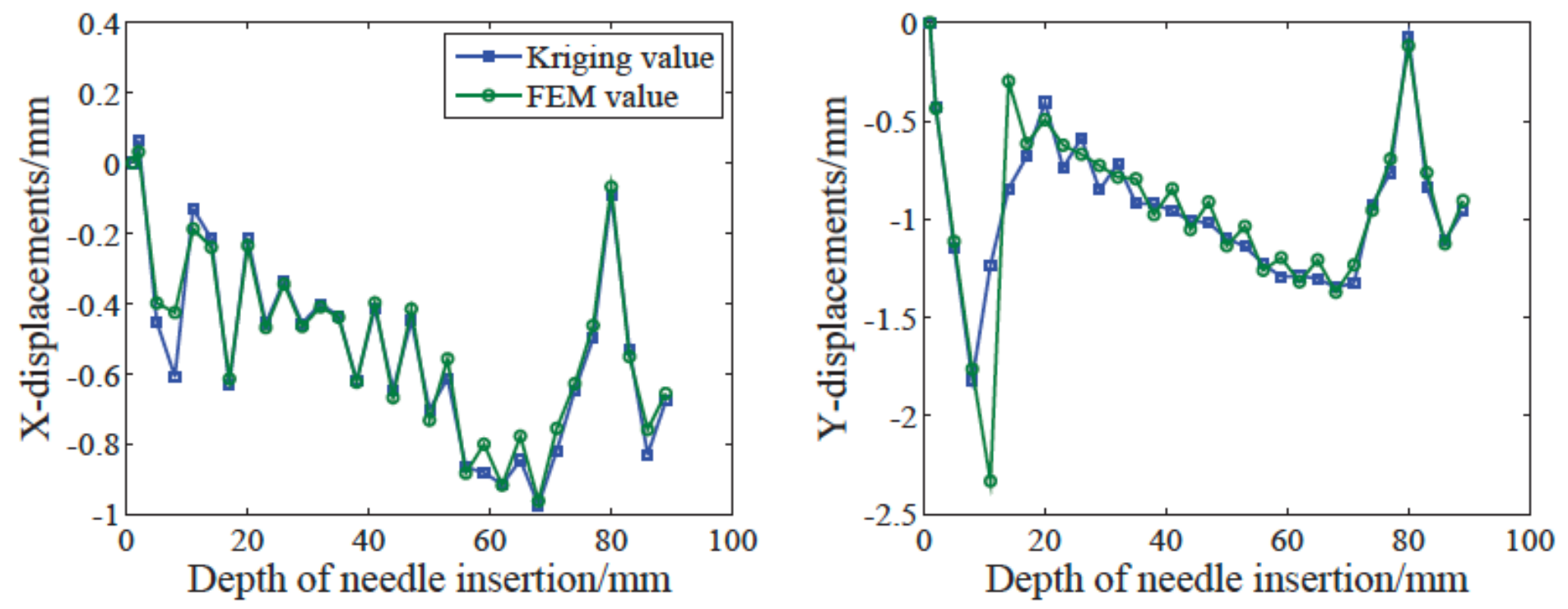

Figure 6

Kriging predictions for insertion depth-tissue displacement curve (N2) 

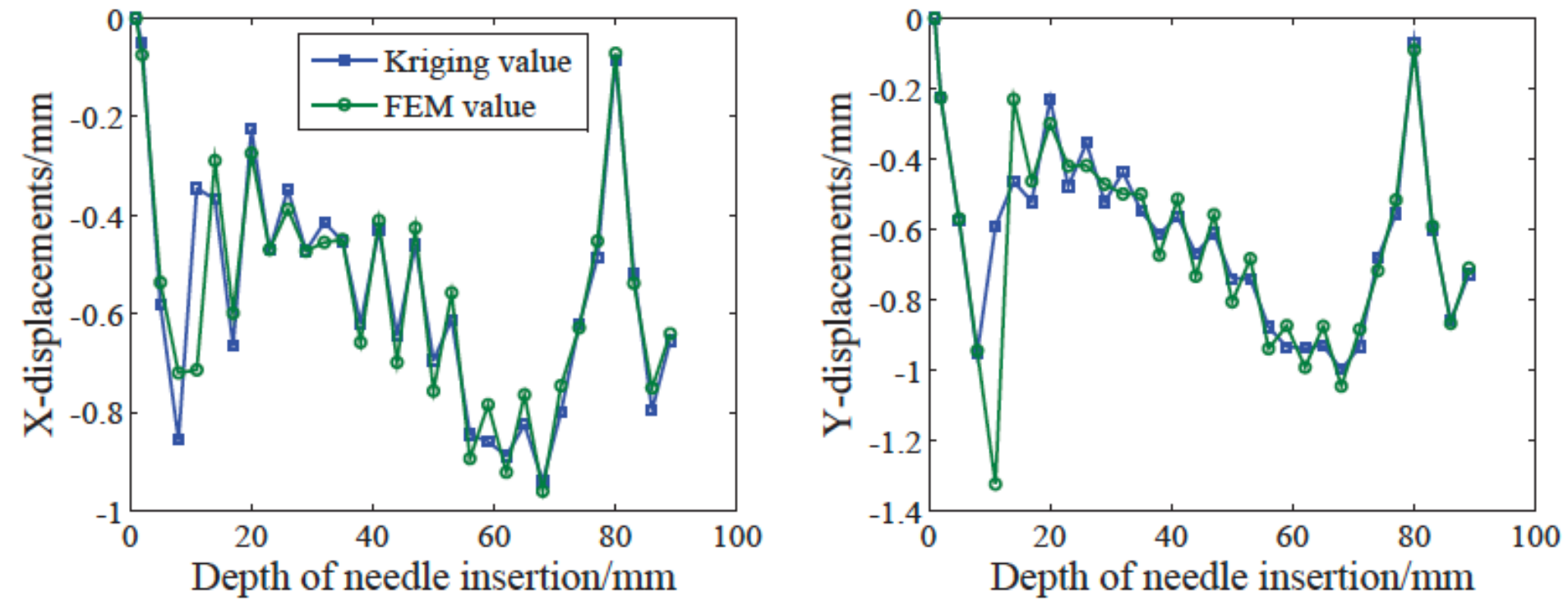

Figure 7

Kriging predictions for insertion depth-tissue displacement curve (N8)
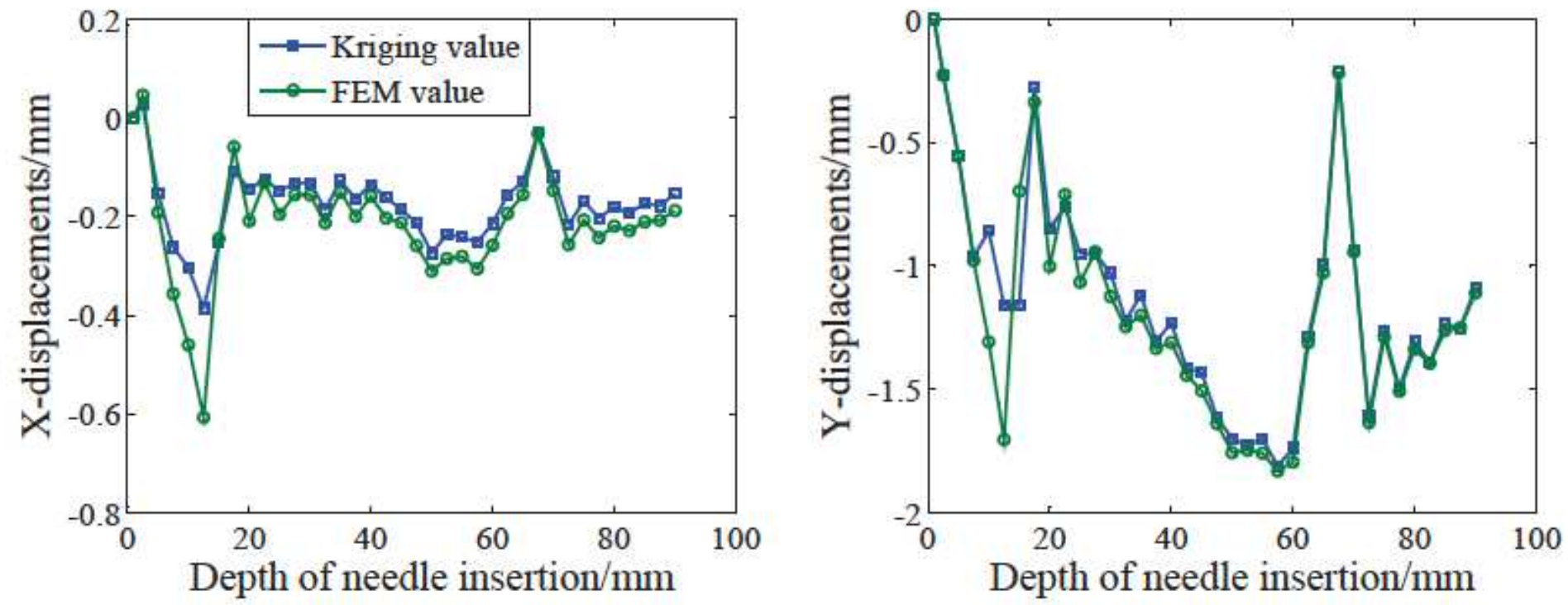

Figure 8

Kriging predictions for insertion depth-tissue displacement curve (N3) 